\title{
Cubic Spline Interpolation Reveals Different Evolutionary Trends of Various Species
}

\author{
Zhiqiang $\mathrm{Li}^{1}$ and Peter Z. Revesz ${ }^{1, a}$ \\ ${ }^{1}$ Department of Computer Science, University of Nebraska-Lincoln, Lincoln, NE, 68588, USA
}

\begin{abstract}
Instead of being uniform in each branch of the biological evolutionary tree, the speed of evolution, measured in the number of mutations over a fixed number of years, seems to be much faster or much slower than average in some branches of the evolutionary tree. This paper describes an evolutionary trend discovery algorithm that uses cubic spline interpolation for various branches of the evolutionary tree. As shown in an example, within the vertebrate evolutionary tree, human evolution seems to be currently speeding up while the evolution of chickens is slowing down. The new algorithm can automatically identify those branches and times when something unusual has taken place, aiding data analytics of evolutionary data.
\end{abstract}

\section{Introduction}

Whereas evolutionary biologists in the past could be satisfied with piecing together an evolutionary tree of related species, now they can ask deeper questions, such as when was the evolutionary change most rapid or slow in any branch of the evolutionary tree [11]. In the past, evolutionary biologists could make only relatively subjective statements about the speed of evolution. However, the DNA data available today in many genome databases [5], [13] for an increasing number of living species and even from ancient DNA from fossils enables modern evolutionary biologists to make more precise and measurable statements about the speed of evolution. This is because the speed of biological evolution from an ancestor species to a descendant species can be measured in the number of genetic mutations.

Data analytics has the potential to make many fascinating discoveries about the evolutionary trends and their causes. Such a data analytics would bring together evolutionary biologists and data scientists. Towards this goal, we describe in this paper a novel evolutionary trend discovery (ETD) algorithm. The ETD algorithm estimates the different trends of evolution for various branches of the evolutionary tree. Our work already brings together cubic spline interpolation from numerical analysis, phylogenetic tree algorithms, and evolutionary biology.

This paper is organized as follows. Section 2 presents some related work. Section 3 describes a method to find evolutionary trends using the fossil record-based age estimates of ancestral species. Section 4 describes the experimental results. Finally Section 5 gives some conclusions and directions for future work

\section{Related work}

Given the genes of a set of related species, a hypothetical evolutionary tree, also called a phylogenetic tree, can be constructed using several different algorithms.

The UPGMA [12] and the Neighbor Joining (NJ) [10] algorithms are the most commonly used phylogenetic tree algorithms. The maximum likelihood method is also well known, although it less frequently used that UPGMA and Neighbor Joining because it requires more computational time. The Common Mutations Similarity Matrix (CMSM) algorithm of Revesz [6], the Incremental Phylogenetics by Repeated Insertions (IPRI) algorithm of Revesz and $\mathrm{Li}$ [4], and Wang's method [14] are some recently proposed phylogenetic tree algorithms. Many phylogenetic tree algorithms are reviewed in the textbooks [1]-[3].

The phylogenetic tree algorithms generate for a given set of genes of a set of related species a common ancestor/root node as well as internal nodes that correspond to the ancestral forms of various branches in the evolutionary tree. Moreover, the algorithms also associate with the root and each internal node an estimated gene (DNA sequence) based on all the descendant species.

There is a strong relationship between the biological classification of species and their evolutionary tree. In fact, the biological classification is often updated to match closer the constantly improving understanding of biological evolution.

For example, Table 1 lists the biological classification of fourteen vertebrate species. A phylogenetic tree generated for these fourteen species

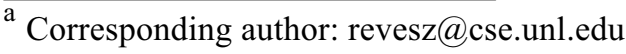


Table 1. The biological classification of forteen vertebrate species

\begin{tabular}{|c|c|c|c|c|}
\hline Species & Phylum & Class & Order & Family \\
\hline Human (Homo sapiens) & Chordata & Mammalia & Primates & Hominidae \\
\hline Cattle (Bos taurus) & Chordata & Mammalia & Cetartiodactyla & Bovidae \\
\hline Dog (Canis familiaris) & Chordata & Mammalia & Carnivora & Canidae \\
\hline Brown rat (Rattus norvegicus) & Chordata & Mammalia & Rodentia & Muridae \\
\hline Mouse (Mus musculus) & Chordata & Mammalia & Rodentia & Muridae \\
\hline Hamster (Mesocricetus auratus) & Chordata & Mammalia & Rodentia & Cricetidae \\
\hline Chicken (Gallus gallus) & Chordata & Aves & Galliformes & Phasianidae \\
\hline Japanese quail (Coturnix japonica) & Chordata & Aves & Galliformes & Phasianidae \\
\hline African clawed frog (Xenopus laevis) & Chordata & Amphibia & Anura & Pipidae \\
\hline Japanese puffer fish (Takifugu rubripes) & Chordata & Actinopterygii & Tetraodontiformes & Tetraodontidae \\
\hline Estuary cod fish (Epinephelus coioides) & Chordata & Actinopterygii & Perciformes & Serranidae \\
\hline Ricefish (Oryzias melastigma) & Chordata & Actinopterygii & Beloniformes & Adrianichthyidae \\
\hline Japanese ricefish (Oryzias latipes) & Chordata & Actinopterygii & Beloniformes & Adrianichthyidae \\
\hline Zebrafish (Danio rerio) & Chordata & Actinopterygii & Cypriniformes & Cyprinidae \\
\hline
\end{tabular}

using the Common Mutations Similarity Matrix algorithm of Revesz [6] is shown in Figure 1.

The biological classification in Table 1 and the phylogenetic tree in Figure 1 correspond well with each other. In particular, the root, which is node 27, corresponds to the ancestors of vertebrates, the Chordata phylum. Node 24 represents the ancestor of fish, while node 26 represents the ancestor of every other vertebrate. Node 25 represents the ancestor of mammals, and node 21 represents the ancestor of rodents, etc. Biologists have used the extensive fossil record of vertebrates to estimate when each ancestor form existed. For example, the ancestor of all vertebrates is estimated to have lived about 525 million years ago. Some of the other known estimates of evolutionary biologists are listed in Table 2.

Our data analytics method also uses the cubic spline interpolation method from numerical analysis. A review and recent extension of the cubic spline method can be found in [7].

Table 2. Fossil-based age estimates of ancestral species.

\begin{tabular}{|l|l|l|}
\hline $\begin{array}{l}\text { Node } \\
\text { Number }\end{array}$ & Classification & Million Years Ago \\
\hline 15 & Beloniformes & N/A \\
\hline 16 & Galliformes & 85 \\
\hline 18 & Muridae & N/A \\
\hline 21 & Rodentia & 66 \\
\hline 22 & Amphibia & 370 \\
\hline 23 & Primates & 56 \\
\hline 24 & Actinopterygii & 420 \\
\hline 25 & Mammalia & 225 \\
\hline 26 & Non-Actinopterygii & 420 \\
\hline 27 & Chordata & 525 \\
\hline
\end{tabular}

\section{The evolutionary trend discovery algorithm}

In this section we describe our Evolutionary Trend Discovery (ETD) algorithm. The pseudocode of our ETD algorithm is shown below. The ETD algorithm takes as input the following:

1. An evolutionary tree $E$.

2. A function $T$ from internal nodes of $E$ to millions of years ago, where for any internal node $N$, the value of $T(N)$ is the estimated evolutionary time from the root of $E$ to $N$. The root $R$ is always assumed to be at time 0 .

3. A function $A$ from nodes of $E$ to amino acid sequences or DNA sequences.

4. A specific leaf node $L$.

The output of the ETD algorithm is the discovered evolutionary trend function $D$. In our description, the function $D$ is a cubic spline interpolation function based on the combination of the genetic and temporal data that is associated with the path from the root to a leaf $L$. However, in theory, the trend function could be generated by several other numerical interpolation methods. Therefore the cubic spline interpolation is used here as an example of this general idea. Cubic spline interpolation gives an interpolating polynomial that is smoother than some other interpolating polynomials such as Lagrange polynomial and Newton polynomial. 
ALGORITHM ETD(E, T, A, L, D)

1 Find the tree E2 that is the same as E except that pointers from the parents to the children are reversed.

2 Create arrays D1 and D2, and initialize $\mathrm{i}=$ path_length $(\mathrm{L})$;

3 current_node $=$ L;

4 while (current_node $\neq \mathrm{R}$ )

$5 \quad$ D1[i] $=$ T[current_node $]$;

6 mutation_number $=$ Hamming $($ A(current_node $)$, $\mathrm{A}(\mathrm{R})$ );

$7 \quad \mathrm{D} 2[\mathrm{i}]=$ mutation_number;

8 current_node $=$ current_node.next(in E2);

$9 \quad \mathrm{i}=\mathrm{i}-1$;

10 end

$11 \mathrm{D} 1[\mathrm{i}]=\mathrm{T}(\mathrm{R})$;

$12 \mathrm{D} 2[\mathrm{i}]=0$;

13 D = Cubic_Spline(D1,D2);

14 Return D;
In the ETD algorithm we assume that we have available as a subroutine Hamming, which computes the Hamming Distance between two strings, and Cubic_Spline, which finds the cubic spine interpolation function with time D1 and corresponding values D2. The ETD algorithm allows us to investigate the evolutionary trend of a given species of interest using the changes in the number of mutations from the root to the leaf node corresponding to that species.

Example 1. Suppose that the ETD algorithm is called with the parameters where the tree is in Figure 1, the function $T$ is in Table 2, the function $A$ is the amino acids that are returned for each internal node by the CMSM algorithm and for each leaf node in Figure 1 the TERT amino acid (which is discussed in detail in Section 4), and the leaf node is 1. As can be seen in Figure 1, here $\mathrm{L}=$ node 1 and $\mathrm{R}=$ node 27 . The path from $\mathrm{L}$, which

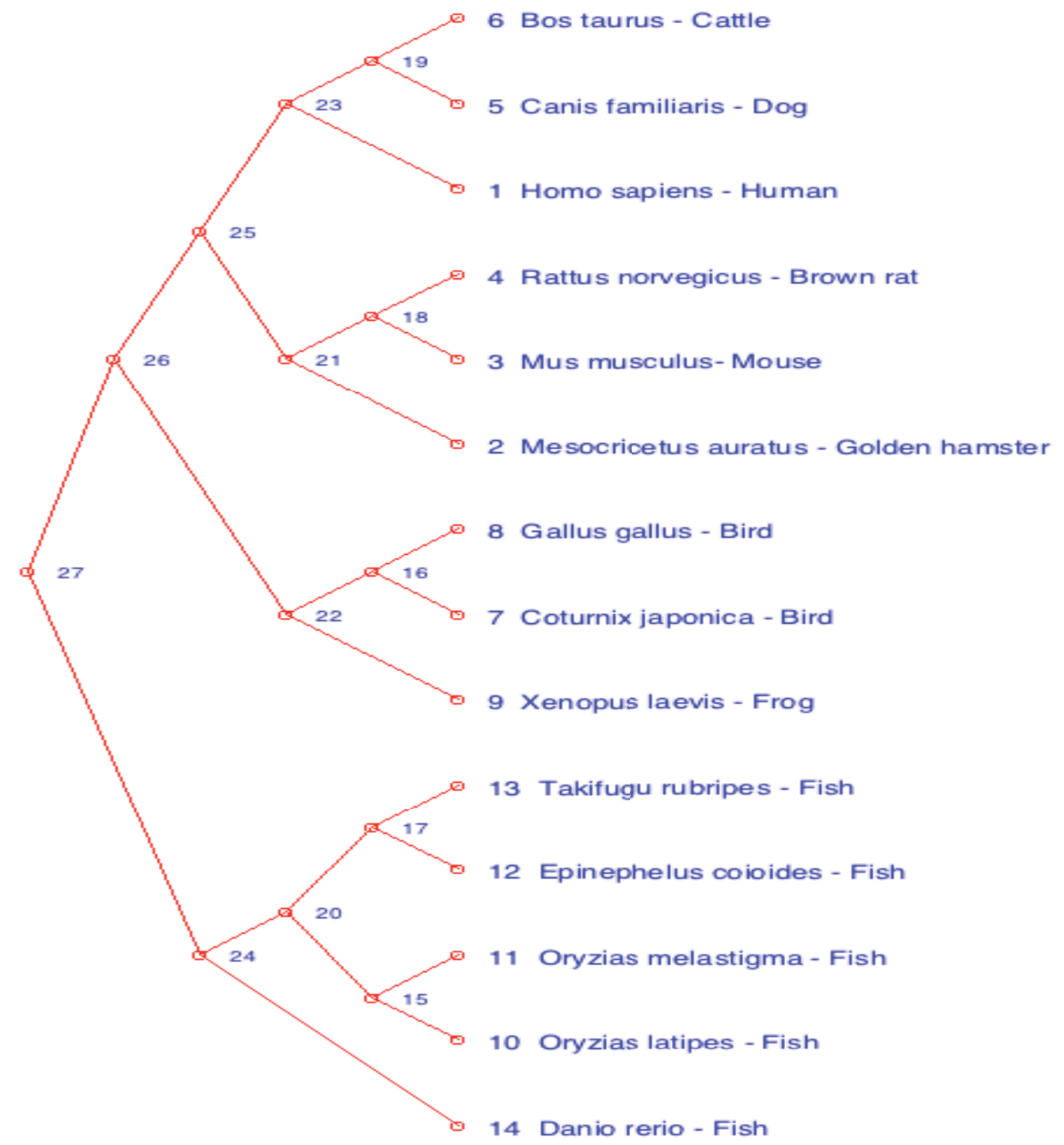

Figure 1. The CMSM phylogenetic tree based on vertebrate telomerase protein data. 
corresponds to humans, to $\mathrm{R}$, which is the ancestral vertebrate, is the following: $1 \rightarrow 23 \rightarrow 25 \rightarrow 26 \rightarrow 27$. Hence the ETD algorithm will work as follows:

$\mathrm{D} 1[4]=\mathrm{T}($ node 1$)$
$\mathrm{D} 2[4]=\operatorname{Hamming}(\mathrm{A}($ node 1$), \mathrm{A}(\mathrm{R}))=83$
$\mathrm{D} 1[3]=\mathrm{T}($ node 23$)$
$\mathrm{D} 2[3]=$ Hamming $(\mathrm{A}($ node 23$), \mathrm{A}(\mathrm{R}))=28$
$\mathrm{D} 1[2]=\mathrm{T}($ node 25$)$
$\mathrm{D} 2[2]=$ Hamming $(\mathrm{A}($ node 25$), \mathrm{A}(\mathrm{R}))=11$
$\mathrm{D} 1[1]=\mathrm{T}($ node 26$)$
$\mathrm{D} 2[1]=$ Hamming $(\mathrm{A}($ node 26$), \mathrm{A}(\mathrm{R}))=4$
$\mathrm{D} 1[0]=\mathrm{T}($ node 27$)$
$\mathrm{D} 2[0]=0$
Build a cubic spline that satisfies $\mathrm{D} 2=\mathrm{D}(\mathrm{D} 1)$.

\section{Experimental results}

As an example, we build an evolutionary tree based on the telomerase (TERT) protein family using the CMSM algorithm. Telomerase help protect eukaryote chromosomes during duplication and is generally present protein in eukaryotes. From the website http://telomerase.asu.edu we obtained 14 vertebrate telomerase proteins as our input data. After alignment, the length of each amino acid sequence was 1353. Figure 1 shows the evolution tree from CMSM.

We evaluate our evolutionary trend discovery algorithm using as test TERT data related to human and chicken evolution. Figure 2 shows the cubic spline interpolation results for both humans and chickens. Each unit on the $\mathrm{x}$ axis in Figure 2 is 1 million years. Both the human and the chicken evolutionary trend functions indicate that the overall number of mutations is increasing with time but at different rates. There are some small periods that can be considered errors in the
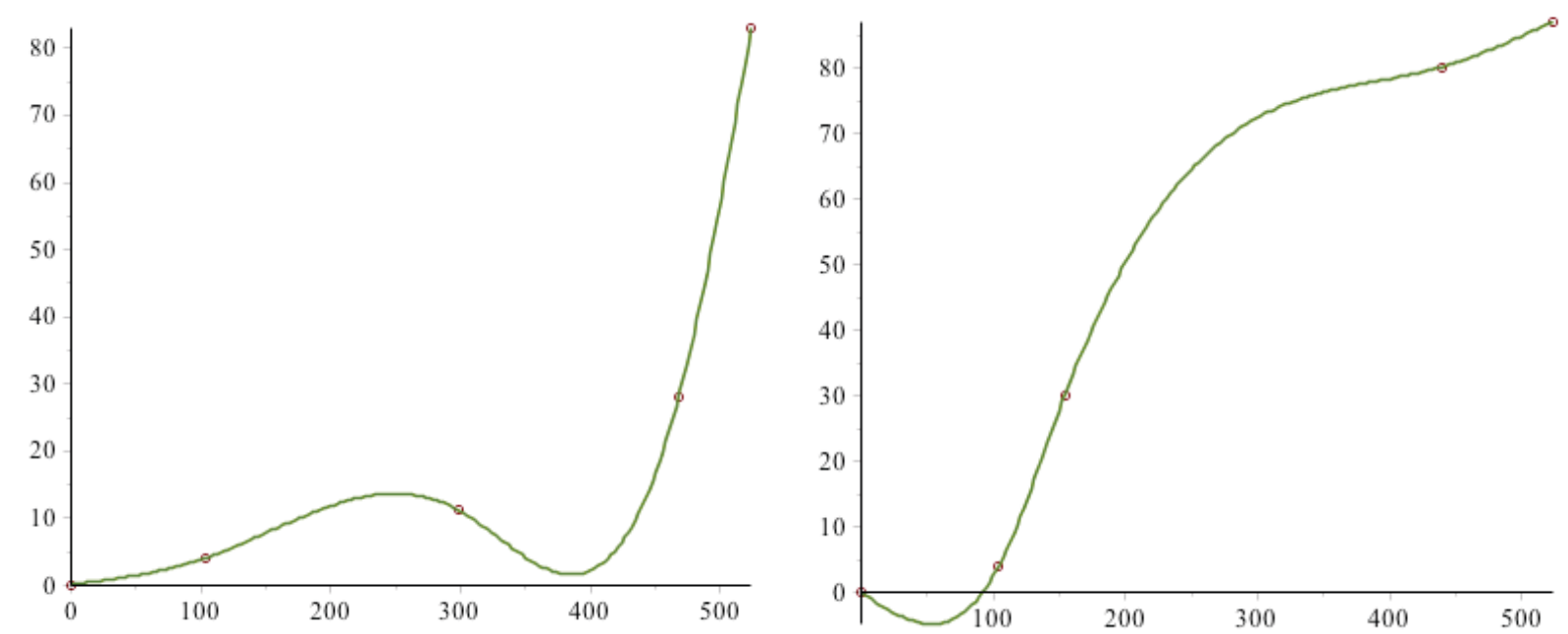

Figure 2. Cubic spline interpolation of the number of evolutionary mutations for human (left) and chicken (right). The $x$-axis is already known millions of years since the common ancestor of all vertebrates, and the y-axis is the number of mutations in the TERT proteins.

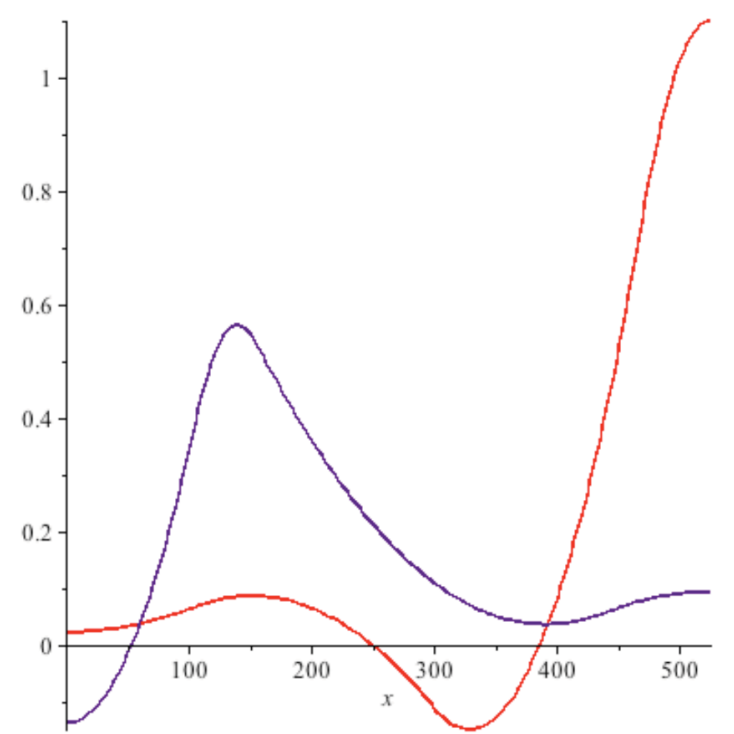

Figure 3. The above figure shows the first derivatives of the functions in Figure 2. The red curve shows the evolutionary trend function associated with humans and the purple curves shows the evolutionary trend function associated with chickens. 
interpolation because the number of mutations should always increase. These blips of errors non-withstanding, the overall trends seem quite reasonable.

In order to check better the evolutionary trends, we also draw the curves of the first derivatives for the evolutionary trend functions as shown in Figure 3. The red curve stands for human evolution, and the purple curve represents chicken evolution from an ancestral vertebrate that lived around 500 million years ago. Figure 3 suggests that the evolution of humans involved a speeding up of the rate of evolutionary mutations. In contrast, the rate of evolutionary mutations slowed down for chickens around 200 million years ago. This result agrees with our intuition with biological evolution as birds have evolved from dinosaurs millions of years ago, and mammals have evolved more recently. There seems to be a more rapid evolution at the beginning of the appearance of radically new forms of species and adaptations to new modes of living, such as flying for birds, and then a decline in the rate of mutations and adaptations after a period of establishment of the new form. It would be interesting to check whether this pattern also appears when considering other genome or protein families and other examples of vertebrates from the mammalian and bird phyla.

\section{Conclusions and future work}

We plan to apply the ETD algorithm to other protein and genome families for both eukaryotes and bacteria. In the ETD algorithm, we also plan to use other estimated time function $T$. Some possibilities include the estimates obtained by the UPGMA algorithm that returns not only an evolutionary tree but also a time estimate for each internal node of the tree. Many other phylogenetic tree algorithms also a time function that may be useful. It remains to be seen which of these estimates is the best and what is the degree of consistency in the results when using all of these different estimates of $T$. The estimating of the time function $T$ by some method is especially important in the case of species that do not have available as extensive fossil records as for the vertebrates.

In addition, in the future more complex data analytics would need to correlate the overall evolutionary trends with significant known events in the history of the earth, such as gradual changes in the atmospheric concentrations of carbon dioxide, oxygen and water vapor, temperature changes, water elevation changes etc. These may enable a deeper data analytics that identifies the significant factors that drive the speed of evolution.

\section{References}

1. D. Baum and S. Smith, Tree Thinking: An Introduction to Phylogenetic Biology, (Roberts and Company Publishers, 2012)

2. B.G. Hall, Phylogenetic Trees Made Easy: A How to Manual, 4th edition, (Sinauer Associates, 2011)

3. P. Lerney, M. Salemi, and A.-M Vandamme, editors. The Phylogenetic Handbook: A Practical Approach to Phylogenetic Analysis and Hypothesis Testing, 2nd edition, (Cambridge University Press, 2009)

4. P.Z. Revesz and Z. Li, "Incremental phylogenetics by repeated insertions: An evolutionary tree algorithm," International Journal of Biology and Biomedical Engineering, 10, 1, 148-158, (2015)

5. P.Z. Revesz, Introduction to Databases: From Biological to Spatio-Temporal, (Springer, New York, 2010)

6. P.Z. Revesz, "An algorithm for constructing hypothetical evolutionary trees using common mutations similarity matrices," Proc. 4th ACM International Conference on Bioinformatics and Computational Biology, 731-734, (ACM Press, New York, 2013)

7. P.Z. Revesz, "A recurrence equation-based solution for the cubic spline interpolation problem," International Journal of Mathematical Models and Methods in Applied Sciences, 9, 1, 446-452, (2015)

8. P.Z. Revesz and T. Triplet, "Classification integration and reclassification using constraint databases," Artificial Intelligence in Medicine, 49, 2, 79-91, (2010)

9. P.Z. Revesz and T. Triplet, "Temporal data classification using linear classifiers," Information Systems, 36, 1, 30-41, (2011)

10. N. Saitou and M. Nei, "The neighbor-joining method: A new method for reconstructing phylogenetic trees," Molecular Biological Evolution, 4, 406-425, (1987)

11. M. Shortridge, T. Triplet, P.Z. Revesz, M. Griep, and R. Powers, "Bacterial protein structures reveal phylum dependent divergence," Computational Biology and Chemistry, 35, 1, 24-33 (2011)

12. R.R. Sokal, and C. D. Michener, "A statistical method for evaluating systematic relationships," University of Kansas Science Bulletin, 38, 14091438, (1958)

13. T. Triplet, M. Shortridge, M. Griep, J. Stark, R. Powers, and P.Z. Revesz, "PROFESS: A protein function, evolution, structure and sequence database," Database -- The Journal of Biological Databases and Curation, (2010)

14. S. Zhang and T. Wang, "A new distance-based approach for phylogenetic analysis of protein sequences," International Journal of Biology and Biomedical Engineering, 3, 3, 35-42, (2009) 